\title{
Magnetic and quadrupolar ordering in $\mathrm{TmNi}_{2} \mathrm{~B}_{2} \mathrm{C}$
}

\author{
N.H. Andersen ${ }^{\mathrm{a}, *}$, J. Jensen ${ }^{\mathrm{b}}$, T.B.S. Jensen ${ }^{\mathrm{a}}$, R. Pinholt ${ }^{\mathrm{a}}$, M.V. Zimmermann ${ }^{\mathrm{c}}$, \\ K. Nørgaard Toft ${ }^{\mathrm{a}}$, A.B. Abrahamsen ${ }^{\mathrm{a}}$, P. Hedegård ${ }^{\mathrm{b}}$, P. Vorderwisch $^{\mathrm{d}}$, P. Canfield ${ }^{\mathrm{e}}$ \\ ${ }^{a}$ Materials Research Department, Risø National Laboratory, DK-4000 Roskilde, Denmark \\ ${ }^{\mathrm{b}}$ Niels Bohr Institute, Ørsted Laboratory, Univeristy of Copenhagen, Universitetsparken 5, DK-2100 Copenhagen Ø, Denmark \\ ${ }^{\mathrm{c}}$ Hamburger Synchrotronstrahlungslabor HASYLAB at Deutsches Elektronen Synchrotron DESY, Notkestrasse 85, 22603 Hamburg, Germany \\ ${ }^{\mathrm{d}}$ Berlin Neutron Scattering Center BENSC, Hahn-Meitner Institute, 14109 Berlin, Germany \\ ${ }^{\mathrm{e}}$ Ames Laboratory and Department of Physics and Astronomy, Iowa State University, Ames, Iowa 50011, USA
}

\begin{abstract}
We present neutron and high-energy synchrotron X-ray diffraction studies to show that the anomalous antiferromagnetic (AF) phase diagram of $\mathrm{TmNi}_{2} \mathrm{~B}_{2} \mathrm{C}$ in an applied field along [ 100$]$ is governed by a quadrupolar ordering of the Tm ions. The ordering is revealed by a distortion of the lattice with the Tm ions displaced along the $c$-axis and modulated with the same wave vector $\boldsymbol{Q}_{\mathrm{A}}=(0.484,0,0)$ as the $\mathrm{AF}$ phase induced by fields larger than $\approx 10 \mathrm{kOe}$. In zero field, the quadrupolar ordering temperature is $T_{\mathrm{Q}} \cong 13.5 \mathrm{~K}$ but increases to about $20 \mathrm{~K}$ in a field of $100 \mathrm{kOe}$. The Tm displacements are also significantly enhanced, by a factor of 10 at $60 \mathrm{kOe}$. A model is presented that accounts for the quadrupolar as well as the low-field $\boldsymbol{Q}_{\mathrm{F}}=(0.94,0.94,0)$ and high-field $\boldsymbol{Q}_{\mathrm{A}}=(0.483,0,0)$ AF ordering previously observed in this compound.
\end{abstract}

(C) 2006 Elsevier B.V. All rights reserved.

PACS: 74.70.Dd; 75.25+z; 75.80. + q

Keywords: Magnetic and quadrupolar ordering; $\mathrm{TmNi}_{2} \mathrm{~B}_{2} \mathrm{C}$; Neutron and synchrotron X-ray diffraction; Superconductivity

\section{Introduction}

The $\mathrm{RNi}_{2} \mathrm{~B}_{2} \mathrm{C}(\mathrm{R}=$ rare earth $)$ compounds have attracted much attention because superconductivity coexists with different types of magnetic ordering at comparable critical temperatures for $\mathrm{R}=\mathrm{Tm}, \mathrm{Ho}, \mathrm{Er}$ and $\mathrm{Dy}$, and their layered tetragonal structure resembles that of the high-temperature superconductors [1]. In $\mathrm{TmNi}_{2} \mathrm{~B}_{2} \mathrm{C}$, superconductivity is found below $T_{\mathrm{c}}=11 \mathrm{~K}$, and neutron diffraction studies have shown that it orders below $T_{\mathrm{N}}=1.8 \mathrm{~K}$ in zero field with an incommensurable antiferromagnetic (AF) structure having modulation vector $\boldsymbol{Q}_{\mathrm{F}}=(0.094,0.094,0)$ and Ising-like magnetic moments oriented along the $c$-axis [2]. Another incommensurable $\mathrm{AF}$ structure with modulation vector $\boldsymbol{Q}_{\mathrm{A}}=(0.483,0,0)$ is observed when a magnetic field larger than approximately

\footnotetext{
${ }^{*}$ Corresponding author. Tel.: +4546774711 ; fax: +4546775758.

E-mail address: niels.hessel@risoe.dk (N.H. Andersen).
}

$10 \mathrm{kOe}$ is applied along the $a$-axis [3,4]. This AF phase is also found in other magnetic $\mathrm{RNi}_{2} \mathrm{~B}_{2} \mathrm{C}$ compounds, but in $\mathrm{TmNi}_{2} \mathrm{~B}_{2} \mathrm{C}$ the magnetic field dependence is peculiar because the transition temperature $T_{\mathrm{N}}$ increases significantly with field, indicating that additional forces than the conventional crystal field (CF) and magnetic interactions act on the magnetic moments. This motivated a search for effects of magnetoelastic and quadrupolar couplings by studies of the associated lattice distortions. The search for a likely candidate for the displacement mode was stimulated by the observation that the electron-phonon interaction is found to be particularly large at Fermi surface nesting vectors close to $(0.5,0,0)$ or $(0,0.5,0)$ in the $\mathrm{R}=\mathrm{Y}$ and $\mathrm{Lu}$ systems [5] and causes softening of the acoustic and optical $\Delta_{4}$-phonon branches (phonons polarized transversely along the $c$-axis) [6]. In order to understand the ordering properties a theoretical model including magnetic exchange and dipolar couplings, $\mathrm{CF}$, magnetoelastic and quadrupolar couplings is established and shown to account for the experimental data. 


\section{Experimental details and results}

The single crystals used in the experiments were grown by a flux method as described in Ref. [7]. They have irregular shapes with dimensions $2 \times 2 \times 0.2 \mathrm{~mm}^{3}$ and $4 \times$ $4 \times 0.8 \mathrm{~mm}^{3}$ for the neutron and X-ray experiments, respectively. The neutron diffraction experiments were carried out in the $a-b$ scattering plane, the synchrotron experiment in the $a-c$ plane. In both experiments, the $a$-axis was aligned along the magnetic field in horizontal cryomagnets. Geometrical constraints required a field offset of $5^{\circ}$ in the synchrotron experiment. The neutron diffraction studies of the magnetic ordering were performed at the BER-II reactor at BENSC, Hahn-Meitner Institut, and at Risø's DR3 reactor (see Refs. [3,4] for more details). The magnetoelastic strain field was studied at the BW5 highenergy beam line at HASYLAB using $100 \mathrm{keV}$ photons and $\mathrm{Si} / \mathrm{Ge}$ gradient crystals as monochromator and analyzer (see Ref. [8] for details). Integrated intensities were recorded along $\boldsymbol{a}^{*}$ and $\boldsymbol{c}^{*}$ at $(0.484,0,8)$ and at the $(0,0,8)$ Bragg peak, which allow determination of the superstructure peak position and a displacement field consistent with the $\Delta_{4}$-phonon mode. The neutron diffraction scattering data of the magnetic order parameter obtained in the $\boldsymbol{Q}_{\mathrm{A}}$-phase at magnetic fields of 21, 40 and $55 \mathrm{kOe}$ are shown in Fig. 1a. Results obtained in the lowand zero-field $\boldsymbol{Q}_{\mathrm{F}}$ phase and for more field values may be found in Refs. $[3,4,8]$. Fig. 1b contains the results of the integrated X-ray scattering intensity for selected field values (cf. Ref. [8] for more field values). The peak position was found to stay fixed at $\boldsymbol{Q}_{\mathrm{A}}=(0.484(2), 0,0)$ within experimental error. The X-ray intensity is completely dominated by the Tm cross-section and is proportional to the square of the displacement amplitude along the scattering vector. Since there is essentially no scattering intensity at $(0.484,0,0)$ it is concluded that the polarization of the displacement is along the $c$-axis. By intensity comparison of the $(0.484,0,8)$ and $(0,0,8)$ peaks, the strain amplitude has been estimated to be $\approx 10^{-2} \AA$ for a scattering intensity of 200 . However, the experimental configuration and the irregular crystal shape result in large and rather uncertain absorption corrections that restrict the accuracy to an order of magnitude estimate.

\section{Theoretical model and analyses}

Early experimental studies of the magnetic properties of $\mathrm{TmNi}_{2} \mathrm{~B}_{2} \mathrm{C}$ by magnetization [7] and by neutron diffraction in fields up to $18 \mathrm{kOe}$ have been analyzed within a magnetic model that included $\mathrm{CF}$, magnetic exchange and dipolar couplings [3]. Consistent with the Fermi surface nesting it was assumed that the $\boldsymbol{Q}_{\mathrm{A}}$ phase would be the stable one also at low fields if the system was in the normal phase, but because the superconducting electrons were more strongly effected by the superzone energy gaps at $\boldsymbol{Q}_{\mathrm{A}}$ than at $\boldsymbol{Q}_{\mathrm{F}}$, the latter phase was stabilized until $H_{\mathrm{c} 2}(0) \approx 20 \mathrm{kOe}$ is approached. However, the extension to a field of $60 \mathrm{kOe}$ clearly showed that the magnetic $\boldsymbol{Q}_{\mathrm{A}}$ phase becomes progressively more stable with increasing field. This highly unusual behavior that an $\mathrm{AF}$ order is stabilized by a uniform field and that a magnetoelastic distortion with wave vector $\boldsymbol{Q}_{\mathrm{A}}$ extends to temperatures far beyond $T_{\mathrm{N}}$, gave evidence that magnetoelastic couplings associated
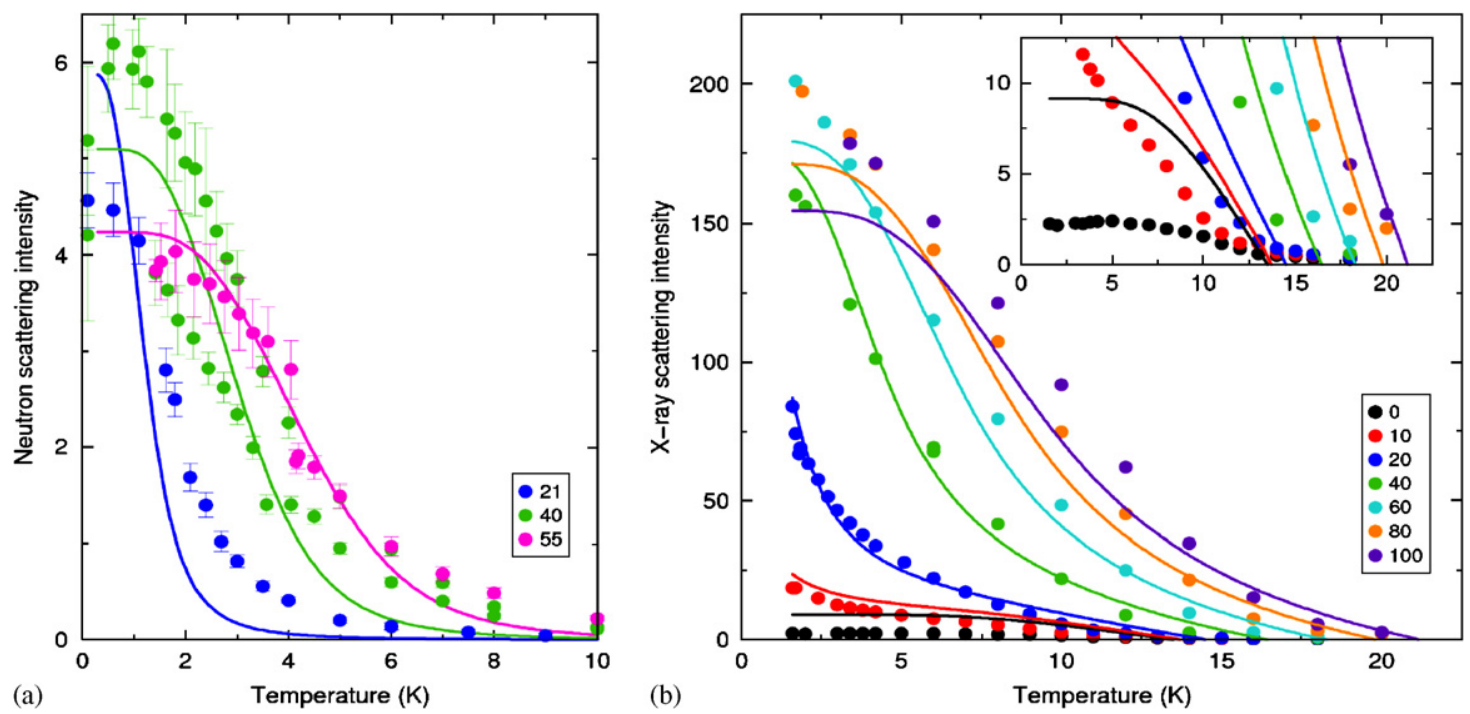

Fig. 1. (a) Magnetic scattering intensity as a function of temperature in the $\boldsymbol{Q}_{\mathrm{A}}$ phase measured at $(0.483,0,0)$ for different magnetic fields (in kOe). The intensity is proportional to and may be converted to the square of the ordered magnetic moment along the $c$-axis. Two different data sets are presented at $40 \mathrm{kOe}$ and their scatter indicates the accuracy by which the absolute value of the magnetic moment is obtained. The solid lines are the result of the model calculations (see the text). Here $5 \mu_{\mathrm{B}}$ corresponds to a neutron scattering intensity of 6 . (b) Synchrotron X-ray diffraction data of the magnetoelastic displacement field measured at $(0.484,0,8)$ for different values of the magnetic field (in kOe). The scattering intensity is proportional to the square of the displacement amplitude in the $c$ direction, and the solid lines are the result of the model calculations (see text). The insert shows an expansion of the lowintensity part of the same results. 
with quadrupolar ordering are essential interactions in $\mathrm{TmNi}_{2} \mathrm{~B}_{2} \mathrm{C}$.

The theoretical model developed to account for the present magnetic and quadrupolar ordering data includes a purely magnetic part with $\mathrm{CF}$, magnetic exchange and classical dipole-dipole interaction, and additional contributions from direct quadrupole-quadrupole interactions, magnetoelastic couplings and elastic strain components to fourth order. The fourth-order strain is necessary because the second-order elastic constant is expected to be small due to the soft $\Delta_{4}$-phonon mode. The purely magnetic part is well characterized from inelastic neutron scattering and magnetization data. The remaining five model parameters are determined by comparison with the present experimental data. A thorough account for the model, the meanfield calculations, the model parameters and how they are determined, is presented elsewhere [8].

The comparison between the model calculations and the experimental data is shown in Fig. 1. At zero temperature, the model predicts a magnetic transition from the $\boldsymbol{Q}_{\mathrm{F}}$ to the $\boldsymbol{Q}_{\mathrm{A}}$ phase at $17 \mathrm{kOe}$ to be compared with the experimental value of $10-15 \mathrm{kOe}$. Fig. 1a shows the comparison between the neutron scattering intensity measured at $(0.483,0,0)$ and the square of the calculated amplitude of the $\boldsymbol{Q}_{\mathrm{A}}$ magnetization mode, which should be proportional. The agreement is satisfactory. The calculated amplitudes are of the right order of magnitude and the temperature variation of the magnetic order parameter is well reproduced. The maximum amplitude is calculated to occur at the entrance to the $\boldsymbol{Q}_{\mathrm{A}}$ phase just above $17 \mathrm{kOe}$, but is found experimentally to be at $40 \mathrm{kOe}$. Instead of focusing on this discrepancy, we shall emphasize the ability of the model in accounting for the disparate variation of the system properties including the magnetoelastic distortion associated with the quadrupolar ordering shown in Fig. $1 \mathrm{~b}$. Consistent with the experimental analysis the model result is scaled to an X-ray intensity of 200 for a displacement amplitude of the $\boldsymbol{Q}_{\mathrm{A}}$ strain mode equal to $6 \times 10^{-3} \AA$. The agreement is very good, not only for the $T_{\mathrm{Q}}$ transition temperatures, but also for the major part of the intensity variations. The only significant discrepancy is at zero and low magnetic fields in the magnetic $\boldsymbol{Q}_{\mathrm{F}}$ phase, displayed in the insert to Fig. 1b, where the calculated scattering intensity is more than twice as large as the measured one. Part of this discrepancy may be attributed to domain effects. In zero field, two degenerate quadrupolar ordering modes exist at $(0.484,0,0)$ and at $(0,0.484,0)$, but only the former is stable when the magnetic field is applied along the $a$-axis. It is the special properties of the CF level scheme that cause a significant increase of the quadrupole susceptibility with magnetic field and the associated increase of $T_{\mathrm{Q}}$. In the zero-temperature limit, the quadru- pole susceptibility becomes even more sensitive to magnetic field, but it cannot quite account for the low field behavior.

\section{Discussion}

The studies and the analysis indicate that the nonmagnetic electron-phonon system in $\mathrm{TmNi}_{2} \mathrm{~B}_{2} \mathrm{C}$, like in the $\mathrm{Lu}$ compound, is very close to a lattice instability. In $\mathrm{TmNi}_{2} \mathrm{~B}_{2} \mathrm{C}$ the transition occurs because of the additional contributions of a magnetoelastic coupling, which derives from the $\mathrm{CF}$ produced by the displacements of the ions, and the quadrupole-quadrupole interactions that may be mediated by the same charge density wave responsible for the strong electron-phonon interaction at $\boldsymbol{Q}_{\mathrm{A}}$. The associated ordering of the quadrupole moments has the unique consequence that a uniform magnetic field induces a modulated magnetic moment perpendicular to the field. The reason is that the paramagnetic moment induced by an applied field along the $a$-axis is rotated towards the $c$-axis in proportion to the size of the induced quadrupolar anisotropy. A further surprising result is that the RKKYexchange interaction seems to be little influenced by the nesting, because the analysis show that the exchange interaction is much smaller at $\boldsymbol{Q}_{\mathrm{A}}$ than at $\boldsymbol{Q}_{\mathrm{F}}$.

The present discovery has basic consequences for the understanding of the magnetism of borocarbides. It raises several questions, such as: Are similar quadrupolar phases present in the other borocarbides? Is the strong peak at $(0.55,0,0)$ in the exchange constant of $\mathrm{ErNi}_{2} \mathrm{~B}_{2} \mathrm{C}[9,10]$ a consquence of quadrupolar ordering rather than RKKY?

\section{Acknowledgments}

This work is supported by the Danish Technical Research Council via the Framework program on superconductivity, and the Danish Natural Science Council via Dansync and DanScatt. P.C.C is supported by the Director of Energy Research, Office of Basic Energy Science under contract W-7405-Eng.-82.

\section{References}

[1] C. Mazumdar, R. Nagarajan, Curr. Sci. 88 (2005) 83.

[2] B. Sternlieb, et al., J. Appl. Phys. 81 (1997) 4937.

[3] K. Nørgaard, et al., Phys. Rev. Lett. 84 (2000) 4982.

[4] K. Nørgaard Toft, et al., Phys. Rev. B 69 (2004) 214507.

[5] S.B. Dugdale, et al., Phys. Rev. Lett. 83 (1999) 4824.

[6] P. Dervenagas, et al., Phys. Rev. B 52 (1995) R9839.

[7] B.K. Cho, et al., Phys. Rev. B 52 (1995) 3676.

[8] N.H. Andersen et al., Phys. Rev. B 73(2006) R020504.

[9] A. Jensen, et al., Phys. Rev. B 65 (2004) 104527.

[10] J. Jensen, Phys. Rev. B 65 (2002) 140514. 